\title{
Introducing Multiple Interaction Devices to Interactive Storytelling: Experiences from Practice
}

\author{
Ekaterina Kurdyukova, Elisabeth André, and Karin Leichtenstern \\ Augsburg University, Institute for Computer Science \\ Multimedia Concepts and Applications \\ Universitätsstr. 6a, D-86135 Augsburg \\ \{kurdyukova, andre, leichtenstern\} @informatik. uni-augsburg.de
}

\begin{abstract}
Introducing multiple interaction devices into an interactive story provides certain advantages to the system: it fosters collaboration in multi-user settings, increases user involvement, and addresses more perception channels and user senses. It is a challenging task for designers to introduce multiple interaction devices into an interactive storytelling (IS) system. In this paper we report on our experiences with the design and evaluation of an IS system, called ORIENT. The system was created for multi-user settings; it embeds various interaction devices, such as mobile phones, a dance pad, a Wiimote, and RFID tags. We report on user feedback and derive design challenges that should be considered when creating interaction devices for IS systems.
\end{abstract}

\section{Introduction}

Existing interactive storytelling (IS) systems often incorporate a variety of interaction devices. Introducing multiple devices to communicate with a story has certain advantages: they enable multimodal interaction, address different human senses, and intensify user experiences. Moreover, in multi-user settings various interaction devices provide more opportunities for collaboration and foster communication between users.

However, it is a challenging task to design an IS system with multiple interaction devices. First, the devices should harmonically enhance the story. They should contribute to the drama and be logically involved in the story flow. Second, in a multiuser scenario, the interaction devices assigned to the users should support their roles in a story and help them stay in character. Finally, the interaction techniques supported by the devices should be easy to understand and to apply. A sophisticated interaction technique can hinder interaction with the story and spoil user experience.

In this paper we describe an IS system called ORIENT. The interaction with the scene is supported by various interaction techniques that enable speech and gesture input as well as physical and spatial interaction. Below we describe the interaction design for our system and report on insights we gained during user tests conducted with teenagers. From user feedback and experiences with existing IS systems, we derive design challenges that should be considered when creating an IS system. 


\section{Design and Evaluation of ORIENT}

The interactive storytelling system ORIENT was developed within the EU-funded project eCIRCUS [1]. The interaction concept of ORIENT was inspired by innovative techniques and collaboration theories. We describe the main idea of the system, its interaction design, report on user feedback, and highlight design findings and failures.

\subsection{ORIENT Story}

ORIENT was developed for teenagers at an age between 13 and 16 years. The main objective of the system is to teach them empathy with people from other cultures. The system aims at making teenagers aware of cultural differences including habits and customs. To achieve this objective, the teenagers had to go through a scenario simulating their visit to an unfamiliar planet called ORIENT.

The scenario is played by a group of three users; each user takes on the role of a spaceship crew member. Their mission leads them to a small planet ORIENT, which is inhabited by an alien race - the nature loving Sprytes. Since a fictional (and not existing) culture is portrayed in the scenario, the application is flexible and suitable for users with various backgrounds.

The users had to save the Sprytes from an approaching meteorite; otherwise the ORIENT would stop its existence. In order to save the life on ORIENT, the users first had to acquire knowledge of the Spryte's habits and customs, befriend the Sprytes and ultimately cooperate with them. In order to cooperate with the Sprytes the user had to move in space, explore the environment, perform actions, and communicate with the Sprytes.

\subsection{Interaction Design in ORIENT}

Interaction in ORIENT is enabled by a variety of devices. The players stand in front of a large display which shows the scene and characters on the ORIENT planet (see Fig. 1, left). The characters, Sprytes, are talking to the users in a fictional "gibberish" language to convey the idea of a fantasy culture. Due to the "gibberish" language, we did not have to realize different culture-specific versions of ORIENT: ORIENT was supposed to be played by German and British teenagers. A fictional culture ensured that neither German nor British players were familiar with ORIENT's culture. The unknown language complicated the teenagers' mission because they had to find alternative ways of communication. Therefore, we had to identify appropriate devices and interaction techniques which would support other verbal and non-verbal interaction channels rather than natural speech.

For verbal communication, we used keyword spotting. In order to address a Spryte, the children had to pronounce its name. For speech interaction, the teenagers were equipped with a mobile device. Mobile devices convey the metaphor of a "decoder device" similar to those used in science fiction series, such as Star Trek.

Using the decoder metaphor, the mobile devices can also be used to select objects in the story. In this case, keyword spotting would not work: the users do not know the Sprytes' terms. However, the users can apply the decoder to scan real world objects and transmit their identification to the Sprytes. For the decoder device, we used Nokia 

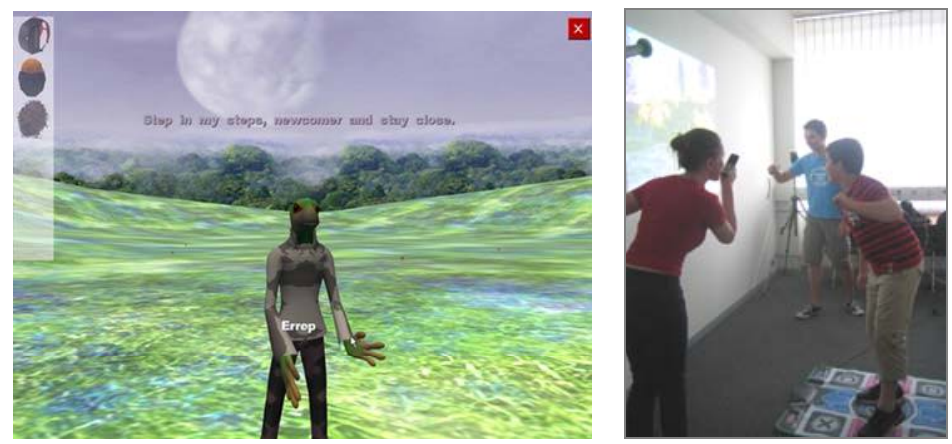

Fig. 1. ORIENT scene (left) and players interacting in a group (right)

6131 NFC, since it supports a built-in microphone for keyword spotting, a built-in NFC-reader for scanning RFID-tagged real world objects and a Bluetooth interface for the transmission of the speech and scanned object to the ORIENT world.

For non-verbal interaction, we chose a set of gestures that the Sprytes could use to communicate: greet, ask, give, and apologize. The users could also adapt and execute these gestures in order to express an action; this was done using a magic stick, the Wiimote. Three-dimensional gesture recognition was performed based on motion data from the Wiimote's accelerometer sensors. In ORIENT, the set of available gestures (and hence, actions) was supposed to be learnt by the users in advance.

Using the decoder device and the magic stick, the users built phrases to communicate with the Sprytes. Every phrase consisted of Subject (Spryte name given by speech), Action (gesture executed with Wiimote) and Objects (scanned RFID tag). In this way, the phrases represented an utterance which was addressed to a particular Spryte, e.g. a question about an object available in the Spryte's world.

Three users participated in the story. Their roles were defined by the interaction devices assigned to them based on a study that explored different multi-user settings for pervasive games [2]. This study showed that a setting where each user is assigned a role via an interaction device with a dedicated function helps organize interactions within a group, fairly distributes the levels of interactivity and avoids dominant users. This setting promoted collaboration among users in a better way than a setting where just one interaction device was given to the whole group or a setting where each group member was equipped with an identical device. Drawing on the findings of this study, roles in ORIENT were assigned as follows. The first role, called Communication Officer, made use of the mobile phone for keyword spotting and RFID scanning of real world objects. The second role, Intelligence Officer, employed the Wiimote for performing the gestures. The third role, Organization Officer, used the dance pad for navigation. Figure 1 (right) shows users interacting with the system. Each of the three interaction devices had a unique function, necessary to achieve the goal of the story.

The final version of the system introduced a mobile-based assistant, called Oracle. The assistant provided the users with help how to proceed with the story. Oracle was implemented on a second mobile device (Nokia N95) and belonged to the user responsible for the navigation with the dance pad. Figure 2 summarizes the interaction devices used in the ORIENT system. 


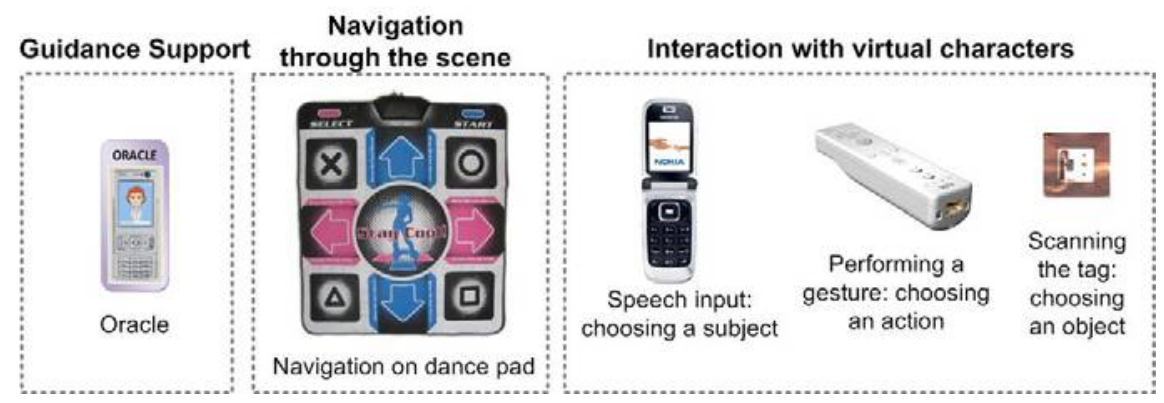

Fig. 2. Interaction devices in ORIENT

\section{Lessons Learnt from User Feedback}

The ORIENT prototype was tested in a lab-based study with 12 teenagers from UK and Germany respectively. Each evaluation lasted about two hours, including device training, interaction with ORIENT, and filling in questionnaires. Below we report on findings based on observations of and feedback from users interacting with ORIENT. We group the insights according to the challenges we faced during the design.

\section{Assure Basic Usability}

User feedback revealed that usability problems may negatively affect IS experience. Even though the children found it challenging to learn Wiimote gestures to communicate with the Sprytes, the number of gestures should be reduced. The users could not remember them and often asked for help. Other sources of confusion were failures of the gesture recognition system. It is crucial to consider the users' cognitive and physical load in order to avoid frustration during interaction while still preserving the challenge of a game-like setting.

\section{Provide Transparent Interaction with the Story}

In order to enhance the transparency of the interaction, the artificial language used to communicate with the Sprytes was oriented by the grammar of natural languages and structured according to the "Subject + Action + Object" pattern. Nevertheless, some users easily confused the subject and the object since in human-human communication, people build phrases more freely. Although both German and British children were able to communicate in our artificial language, more control and assistance from the system would be desirable. Moreover, the borders of interaction should be specified more clearly. For example, the designers of Locating Drama [3] exploit sound to give users a hint about the borders of interaction space. The system is played in a park whereby borders and exits from the "story space" are marked with a certain noise. In IS systems, where users interact using natural language or languages bearing some resemblance to natural languages, such as ORIENT, ideas from prompt design might help show the user how and when they can interact. For example, in FearNot! [4] carefully designed dialogue strategies for characters interacting with children encouraged specific types of follow-up natural language responses from the children and thus reduced the likelihood of unexpected user input. 


\section{Support Unobtrusive Guidance through the Story}

The guidance through the ORIENT story was supported by Oracle, an assistant on the mobile phone. In the case of confusion, the users consulted Oracle. Oracle turned out to be a promising approach for user assistance since it was integrated naturally into the story. Nevertheless it has to be improved to act in a more intelligent way. For example, if the question was formulated incorrectly or did not match the current scene, Oracle just answered "The question is irrelevant". This caused user frustration: the users still did not know how to proceed. Ideally, user guidance should be supported by interaction design. Designers of the Mobile Urban Drama [5] found that audio assistance can significantly help guide the users. However, the guidance should be done unobtrusively, e.g. as "voices in your head" [3]. General design principles, such as affordances, constraints and mappings, may also help guide users without providing lengthy instructions. In ORIENT, users navigated through a scene by stepping on an appropriate pad arrow of a dance pad. Such design bears a resemblance to real-world navigation (even though it does not provide an appropriate mapping for all aspects of real world navigation). Moreover, it only occupied the user's feet, and allowed the user to perform other actions simultaneously.

\section{Assure Immersion through Scene Design}

Prior research has shown that embedding a story in the user's physical environment helps increase user immersion. Designers of Mobile Urban Drama [5] found that the integration of the user's real surroundings - in their case the physical settings of a city - play an important role in user immersion. Their users even had the impression that passers-by participated in the story. Dow et al. showed that an Augmented Reality version of Façade system was perceived as more immersive than a desktop-based version [6]. However, users found it less engaging than the desktop-based version. ORIENT uses audio effects, such as nature sounds (birds, trees) to immerse the users into a green planet scenario. This idea could be developed further to help users "feel" the mood of the scene. In addition, ORIENT allows for bodily activity and manipulation of physical objects, for example, by scanning them using the mobile phone.

\section{Increase Engagement}

The use of popular input devices, such as the Wiimote, made the interaction for the children more enjoyable and contributed significantly to their engagement. Furthermore, the distribution of input devices based on the findings of the study mentioned earlier [2] helped involve all children actively in the story. Every user had to make use of his or her input device to enable communication with the characters. The effect was enhanced by bodily activity and manipulation of physical objects.

\section{Support Story Plot}

In ORIENT, we aimed at smoothly embedding the interaction devices into the story plot. For instance, the decoder device draws on the metaphor of a communication device which fits to the story of a space command mission. Interaction devices and techniques should harmonically match the story plot in order not to break the illusion. For instance, Madame Bovary [7] uses emotional speech for story control. This input modality is obviously more appropriate for the setting and the scenario than input modalities exploiting futuristic-looking technical devices. 


\section{Providing Plausible Interpretation Possibilities for Deficient Input Interpretation}

Dow and colleagues recommend to design communication in an IS in such a way that system failures in understanding human input can be interpreted by users as a proper reaction of the story [6]. This strategy was employed successfully in ORIENT as well. In ORIENT, incorrect user input or failures of the recognition technology could be interpreted by the users as naturally arising communication problems between different cultures.

\section{Conclusion}

Introducing multiple interaction devices is a challenging task for interaction designers. In this paper we reported on our experience with an IS called ORIENT which includes multiple interaction devices and exploits a variety of interaction techniques. The evaluation of the system gave us an insight into the critical challenges of IS system design: interaction devices should be easy to use while maintaining the challenge of a game-like setting, enhance interaction transparency, provide unobtrusive guidance, immerse users in a story, maximize their engagement, support the story plot and leave enough plausible interpretation possibilities for system failures.

Acknowledgments. This work was partially supported by the EC and has been funded by the projects eCIRCUS (IST-4-027656-STP) and IRIS (FP7-ICT-231824).

\section{References}

1. eCIRCUS (IST-4-027656-STP), http://www.e-circus.org/ (last access 09-242009)

2. Leichtenstern, K., André, E.: Studying Multi-User Settings for Pervasive Games. In: Oppermann, R., Eisenhauer, M., Jarke, M., Wulf, V. (eds.) Proc. 11th Int. Conf. on HumanComputer Interaction with Mobile Devices and Services, pp. 190-199. ACM, New York (2009)

3. Parry, N., Bendon, H., Boyd Davis, S., Moar, M.: Locating Drama: A Demonstration of Location-Aware Audio Drama. In: Spierling, U., Szilas, N. (eds.) ICIDS 2008. LNCS, vol. 5334, pp. 41-43. Springer, Heidelberg (2008)

4. Dias, J., Ho, W.C., Vogt, T., Leichtenstern, K., André, E.: I Know What I Did Last Summer: Autobiographic Memory in Synthetic Characters. In: Paiva, A.C.R., Prada, R., Picard, R.W. (eds.) ACII 2007. LNCS, vol. 4738, pp. 606-617. Springer, Heidelberg (2007)

5. Hansen, F.A., Kortbek, K.J., Grønbæk, K.: Mobile Urban Drama - Setting the Stage with Location Based Technologies. In: Spierling, U., Szilas, N. (eds.) ICIDS 2008. LNCS, vol. 5334, pp. 20-31. Springer, Heidelberg (2008)

6. Dow, S., Mehta, M., Harmon, E., MacIntyre, B., Mateas, M.: Presence and Engagement in an Interactive Drama. In: Proc. of the SIGCHI conference on Human factors in computing systems, pp. 1475-1484. ACM, New York (2006)

7. Cavazza, M., Pizzi, D., Charles, F., Vogt, T., André, E.: Emotional Input for Characterbased Interactive Storytelling. In: Decker, Sichman, Sierra, Castelfranchi (eds.) Proc. 8th Int. Conf. on Autonomous Agents and Multiagent Systems, pp. 313-320. IFAAMAS (2009) 